\section{BMJ Open Respiratory Research}

\title{
Integrated diagnostic pathway for patients referred with suspected OSA: a model for collaboration across the primary-secondary care interface
}

\author{
Nikesh Devani (D , ${ }^{1}$ Tom Aslan, ${ }^{2}$ Fiona Leske, ${ }^{1}$ Stephanie K Mansell (D) , ${ }^{1}$ \\ Sarah Morgan, ${ }^{2}$ Swapna Mandal (ib ${ }^{1,3}$
}

To cite: Devani N, Aslan T, Leske F, et al. Integrated diagnostic pathway for patients referred with suspected OSA: a model for collaboration across the primary-secondary care interface. BMJ Open Resp Res 2020;7:e000743. doi:10.1136/ bmjresp-2020-000743

- Additional material is published online only. To view please visit the journal online (http://dx.doi.org/10.1136/ bmjresp-2020-000743).

Received 13 August 2020

Revised 1 October 2020 Accepted 3 October 2020
(A) Check for updates

(C) Author(s) (or their employer(s)) 2020. Re-use permitted under CC BY-NC. No commercial re-use. See rights and permissions. Published by BMJ.

${ }^{1}$ Respiratory Medicine, Royal Free London NHS Foundation Trust, London, UK

${ }^{2}$ Hampstead Group Practice, London, UK

${ }^{3}$ University College London, London, UK

Correspondence to Dr Swapna Mandal; swapnamandal@nhs.net

\section{ABSTRACT}

Background Obstructive sleep apnoea (OSA) presents a major healthcare challenge with current UK data suggesting that only $22 \%$ of individuals have been diagnosed and treated. Promoting awareness and improving access to diagnostics are fundamental in addressing these missing cases and the recognised complications associated with untreated OSA. Diagnosis usually occurs in secondary care with data from our trust revealing long wait times to undertake tests, reach a diagnosis and start treatment. This places a considerable time and emotional burden on the patient and a financial and logistical burden on the hospital.

Methods We introduced an integrated community-based pathway for the diagnosis of OSA. This comprised a monthly clinic run from within a local general practice (GP) supported by a 'virtual multidisciplinary team' run by the hospital specialist team. Prospective collection of process, outcome and patient satisfaction data was compared with traditional hospital-based pathway data collected retrospectively.

Setting A central London teaching hospital and GPs within a local commissioning neighbourhood.

Results Between January 2018 and February 2019, 70 were patients referred and managed along the community pathway. Compared with the hospital pathway, data demonstrated a significant reduction in the time taken: from referral to perform a sleep test (29 vs 181 days, $p<0.0001$ ), to make a diagnosis ( 40 vs 230 days, $p<0.0001$ ) and commence treatment ( 127 vs 267 , $\mathrm{p}<0.0001)$. Patient satisfaction in the community pathway was higher across all domains $(p<0.05)$, fewer hospital outpatient appointments were required and cost estimates suggested an overall saving of up to $£ 290$ could be achieved for each patient.

Conclusion An integrated community-based pathway results in more timely diagnosis of OSA within a local setting while maintaining specialist input from the hospital team. It is favoured by patients and can reduce unnecessary appointments in secondary care.

\section{INTRODUCTION}

Obstructive sleep apnoea (OSA) is a common, treatable condition estimated to affect up to $4 \%$ of middle-aged men and $2 \%$

\section{Key messages}

What is the key question?

- Can a community-based approach to diagnosing obstructive sleep apnoea (OSA) deliver high-quality patient care while affording cost and time efficiencies?

\section{What is the bottom line?}

- A community-based and hospital-led pathway for the diagnosis of OSA allows for provision of care closer to home and results in quicker diagnosis and treatment, this can generate systems cost-savings and is preferred by patients.

Why read on?

- This novel pathway aligns with the National Health Service Long Term Plan and can serve as a framework for other service development pathway projects.

of middle-aged women in the $\mathrm{UK}^{1}$ and up to 1 billion adults globally. ${ }^{2}$ Undiagnosed and untreated OSA presents a significant health challenge with increased risk of cardiovascular and cerebrovascular events ${ }^{3}$ and metabolic complications such as insulin resistance, diabetes and obesity. ${ }^{4}$ Furthermore, individuals with untreated OSA are up to seven times more likely to suffer a motor vehicle accident ${ }^{5}$ with this risk removed when patients are on treatment. ${ }^{6}$ Despite this, estimates from the British Lung Foundation (BLF) suggest that only $22 \%$ of patients with OSA have been diagnosed and treated, resulting in over 1.2 million untreated cases. ${ }^{7}$

Diagnosis and management of OSA requires specialist input and often takes place in secondary care following referral from a primary care clinician, in the UK this is usually a general practitioner (GP) (online supplemental file 1 provides a detailed typical patient pathway). Following review at an outpatient specialist secondary care 
clinic, the patient is required to undertake a sleep study usually in their own home. Sleep study devices can vary from trust to trust but most will monitor oxygen saturation, heart rate and flow of air through the nose during sleep (limited cardiorespiratory polygraphy). After undertaking their sleep study and returning the device, the patient is seen again in clinic to be given their diagnosis and commenced on treatment if appropriate. This current pathway has multiple steps each with the potential to result in a time delay and also places a significant reliance on hospital outpatient clinics. A more responsive and streamlined approach to healthcare delivery was advocated in the National Health Service (NHS) plan $^{8}$ and more recently in the NHS Long Term Plan ${ }^{9}$ with an emphasis on 'care closer to home,' 'removal of unnecessary stages of care' and tests and treatment being 'offered on a one-stop basis'. ${ }^{9}$ In response to this and the pressing need to access the many undiagnosed cases, we developed an integrated community-based hospital-led pathway for the diagnosis of OSA. Our pathway allows a patient to undergo a preliminary assessment and receive a sleep study device at a GP practice close to home but maintains specialist input through a virtual multidisciplinary team (MDT) provided by the sleep and ventilation department based at an NHS Trust. Within this paper, we prospectively evaluate our pathway and report outcomes following its implementation within one GP catchment neighbourhood.

\section{METHODS}

Setting

This new pathway was coordinated by a London-based teaching hospital which provides services to approximately 1.6 million patients principally located within four London boroughs. The specialist sleep and ventilation service at this trust receives in the region of 1500 referrals annually for suspected OSA and also provides care for patients across the spectrum of sleep disordered breathing. Within one of the catchment boroughs, GPs are arranged into collaborative working neighbourhoods based on their geographical location. The new pathway was introduced in one of these neighbourhoods. We used a 'hub and spoke model' to establish a monthly community outreach OSA clinic within a centrally located 'hub' GP practice. This clinic received referrals for patients with suspected OSA directly from other neighbourhood GP practices (the 'spokes'). For the purposes of this pilot, this clinic was staffed by a sleep and ventilation clinical fellow and consisted of a half day session with capacity to review eight patients. To refer a patient, GPs based at any of the other 'spoke' practices within the neighbourhood were required to complete an electronic referral proforma which collected basic patient data as well as a pre-test probability score (STOP-BANG) and an assessment of somnolence (Epworth Sleepiness Scale, ESS). This form was embedded within the practice electronic healthcare portal (EMIS Web, EMIS group, Leeds, UK) and once completed, was sent via a dedicated confidential NHS mail account to the sleep and ventilation team. Referrals were screened by the sleep and ventilation clinical fellow and those with a STOP-BANG $\geq 3$ were accepted and subsequently allocated to an appropriate clinic appointment at the hub practice. Patients with impaired mobility (who would normally require hospitalprovided transport to attend appointments) and those who required externally provided translation services were excluded from this pathway and instead booked into a regular hospital appointment.

At the outreach clinic, the patients underwent a brief consultation and were issued with a portable diagnostic device to allow them to undertake a home sleep study (WatchPAT, Itamar Medical, Keisarya, Israel). Patients were assessed for somnolence and non-respiratory sleep disorders using a comprehensive structured sleep questionnaire (online supplemental file 2). Once the devices and questionnaires had been returned to the hub practice, they were reviewed within a virtual MDT meeting run by the specialist sleep and ventilation team based at the London NHS Foundation Trust. The final diagnosis, suggested management and follow-up arrangements, were then communicated directly to the patient and their registered GP. Those patients requiring treatment or further review were automatically scheduled to attend a secondary care clinic.

\section{Data collection}

We initially assessed the demands faced by the hospitalbased sleep and ventilation service by retrospectively auditing a random sample of referrals to this service between June 2016 and June 2017. This gave us insight into the waiting times, costs and the diagnoses being made in this conventional hospital-based pathway. Furthermore, we undertook a process-mapping exercise in parallel to determine a typical patient pathway from GP referral through to treatment and help identify areas for streamlining particularly when designing the community outreach pathway (online supplemental file 1).

The design of the new pathway was undertaken in collaboration with local GPs, governing members of the Clinical Commissioning Group and the hospital operations team and followed a series of planning meetings with all stakeholders present. Following introduction of the community outreach pathway, prospective evaluation of the service was undertaken over 13 months (January 2018-February 2019). Process measures such as waiting times (from referral to appointment, investigation, diagnosis and treatment) were collected longitudinally and estimates of costs and the final diagnoses made were compiled at the conclusion of pathway implementation. Patient satisfaction was collected using an established questionnaire comprising standardised numerical rating scale (NRS). Patient satisfaction for those attending hospital clinics along the traditional pathway using a similar questionnaire was also collected to serve as a 
comparator. More detail regarding this is provided in the Patient and public involvement section below. Clinician satisfaction was collected using an online survey (Survey Monkey, SVMK, San Mateo, California, USA) distributed to all senior partner GPs based within practices participating in the community OSA pathway.

Cost estimates of each pathway were calculated using the tariffs listed within the NHS National Tariff $2017 / 2018$ workbook and the pay scales according to 'Agenda for Change' (AfC). In the UK, all healthcare professionals (HCPs) aside from doctors and dentists, are remunerated according to a national pay system called AfC. This system allocates pay according to set 'bands' which reflect the knowledge, experience and skills of the staff. There are nine pay bands in the UK and, for example, 'band 7' would be representative of a typical advanced clinical practitioner or senior physiotherapist. More detail on cost breakdowns and assumptions made have been provided in the Results section.

\section{Data analysis}

All results were summarised with appropriate measures of distribution (mean, median, SD) and a Mann-Whitney U test (GraphPad Prism V.7, San Diego, California, USA) was used to compare outcomes between the conventional hospital pathway and the new community-based pathway.

Accuracy and completeness of data was ensured throughout by cross-checking of data entry during MDT meetings and involvement of expert stakeholders when determining pathway costs and process measures. Data were securely stored on NHS Trust computers and the pathway was registered as a service improvement project locally. The Standards for Quality Improvement Reporting Excellence checklist was used when writing the final report. ${ }^{10}$

\section{Patient and public involvement}

Prior to the design of the new pathway, patients attending a hospital sleep and ventilation clinic were invited to complete a structured questionnaire to capture their experiences of the existing service and understand their priorities and preferences when attending an outpatient appointment. This feedback guided the development of the new pathway and informed our outcome measures. Furthermore, patient feedback collected following each monthly hub clinic allowed for continued refinement of the pathway throughout the pilot period. At the conclusion of the pilot period, we conducted semistructured face-to-face interviews with two patients referred along this community pathway. This allowed us to gather more detailed feedback and understand how we might adapt and improve on this pathway to continue to meet the needs of our patients.

Pathway outcomes were not directly disseminated to patients but were made available to access through notices and communications posted within the local GP practices.

\section{RESULTS}

\section{Waiting times and diagnoses}

Ninety-one patients were referred to the community outreach pathway between January 2018 and February 2019; and following initial screening using a pre-existing referral proforma, all were deemed suitable to attend. Baseline demographics of these patients compared with those managed along the hospital pathway are provided in table 1 .

Of these, 70 patients attended a community clinic review and undertook a home sleep study; 21 patients (23\%) did not attend the appointment offered to them. (The average non-attendance rate for the hospital pathway was approximately 33\%). When compared with data obtained from the retrospective analysis of referrals to the conventional hospital-based pathway, waiting times were significantly reduced across all domains (table 2 ).

The final diagnoses reached in the community outreach pathway and conventional hospital pathway are summarised in online supplemental file 3. Twenty-two patients $(31.4 \%)$ in the community pathway were assessed as having moderate or severe OSA or a hypoventilation syndrome on the basis of their study and required specialist treatment. A further 17 patients $(24 \%)$ had mild OSA and a proportion of these with a significant symptom burden were offered ongoing follow-up in a secondary care clinic. Thirty patients $(42.9 \%)$ either had a normal study or one demonstrating only snoring or partial airflow compromise during sleep, and these patients were deemed appropriate for ongoing GP managed care. Similarly, $48 \%$ of patients $(n=49)$ within the hospital pathway had a normal sleep study or one demonstrating only snoring or partial airflow compromise. Of the remaining sampled patients referred along the hospital pathway, 35 (34\%) were assessed as having moderate or severe OSA or a hypoventilation syndrome and $18(17.6 \%)$ as having mild OSA. All patients referred along the hospital pathway attended on average two clinic appointments and waited over 32 weeks to be told their diagnosis compared with 6 weeks in the community pathway group.

\section{Patient and GP satisfaction}

Twenty-seven patients attending the hospital pathway and 61 patients attending the community pathway completed a feedback questionnaire assessing their satisfaction using standardised NRS (ranging from 1 to 5; with 1 reflecting 'not at all satisfied' and 5 representing 'very satisfied'). The results demonstrate significantly higher satisfaction scores across all domains for patients referred to the community pathway compared with a parallel cohort referred along the hospital pathway (table 3). Free text comments reinforced this view and a semistructured interview of one patient (P1) highlighted additional benefits including the notion of a community OSA pathway 'fostering continuity of care in a familiar environment' and 'minimising the need to frequently attend 
Table 1 Demographics of patients managed within the hospital pathway (retrospectively collected data) and community pathway (prospective)

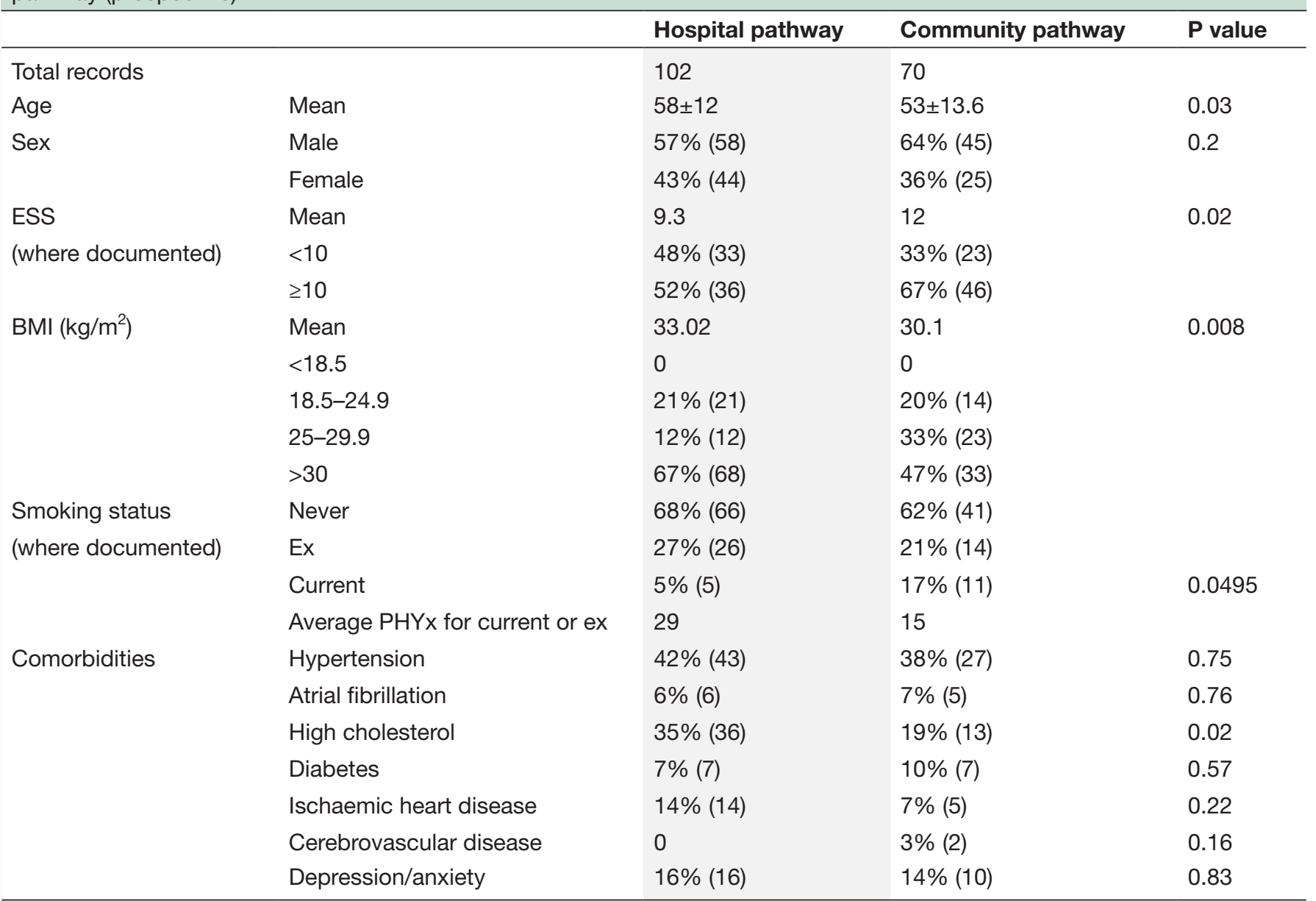

BMI, body mass index; ESS, Epworth Sleepiness Score; PHYx, pack-year history.

hospital clinics which are often a source of anxiety.' At the conclusion of the 13-month observation period, a survey of 10 GPs from participating practices affirmed a preference for a community-based diagnostic approach. Specific comments from those clinicians surveyed commended the 'responsiveness and convenience of the pathway,' 'the simple referral and rapid communication between HCPs that it permits' and 'the stronger relationships it developed between the hospital trust and primary care providers.'

\section{Cost assessment}

From referral to diagnosis made, the community OSA pathway incurred an average estimated overall cost per patient of £442.31 representing a significant cost-saving when compared with $£ 732.81$ for the hospital-based pathway. Table 4 provides a breakdown of costs.

We estimated the cost for each pathway using the tariffs listed within the NHS National Tariff 2017/2018 workbook. The listed tariff for a hospital outpatient

Table 2 Waiting times from referral for hospital and community pathway

\begin{tabular}{|c|c|c|c|}
\hline Measure & Hospital pathway & $\begin{array}{l}\text { Community outreach } \\
\text { pathway }\end{array}$ & $P$ value \\
\hline & \multicolumn{3}{|c|}{ Average time in days (SD) } \\
\hline Time from referral to first outpatient appointment & $113(69.6)$ & $29(16)^{\star}$ & $<0.0001$ \\
\hline Time from referral to sleep study & $181(76)$ & $29(16)^{*}$ & $<0.0001$ \\
\hline Time from referral to patient informed of diagnosis & $229.5(102.8)$ & $39.8(15.9)$ & $<0.0001$ \\
\hline Time from referral to treatment commenced (if appropriate) & $266.9(114.4)$ & $126.8(47.1)$ & $<0.0001$ \\
\hline
\end{tabular}

*For the community outreach pathway, the sleep study was issued at the hub appointment and undertaken by the patient at home that evening. 


\begin{tabular}{llcc} 
& (SD) & & P value \\
\hline The waiting time for today's appointment after being referred by your GP & $3.38(1.6)$ & $4.56(0.8)$ & 0.004 \\
\hline $\begin{array}{l}\text { The waiting time to undergo the sleep study test } \\
\text { The waiting time to be told your diagnosis/the outcome of the sleep }\end{array}$ & $3.53(1.4)$ & $4.67(0.7)$ & $<0.0001$ \\
study test & & $4.53(0.6)$ & 0.004 \\
\hline $\begin{array}{l}\text { The waiting time to be set up with a CPAP device (if applicable) } \\
\text { The distance you had to travel to attend clinic today }\end{array}$ & $3.46(1.6)$ & $4.77(0.5)$ & 0.0035 \\
\hline The waiting time in the clinic to see a member of the team & $3.81(1.4)$ & $4.85(0.4)$ & $<0.0001$ \\
\hline The amount of time you had with the doctor & $4.35(0.9)$ & $4.90(0.3)$ & 0.0001 \\
\hline How would you rate the organisation of the clinic today? & $4.21(1.1)$ & $4.88(0.3)$ & 0.0002 \\
\hline Overall satisfaction & $4.28(1.0)$ & $4.83(0.4)$ & 0.0009 \\
\hline
\end{tabular}

CPAP, continuous positive airway pressure; GP, general practitioner; NRS, numerical rating scale.

appointment is a standard figure which encompasses clinician expenses as well as hospital overheads and ancillary staff support (eg, clinic receptionists, nurses, porters). For a new respiratory patient appointment this is £208 and follow-up £94; using our figure of an average of two appointments before diagnosis in the hospital pathway (one new and one follow-up), this totals £302. Within the community pathway, premises overheads and ancillary support were provided free of charge and hence the cost listed only considers the HCP wage. While during this pilot of the pathway, the clinic was run by a clinical fellow; we have modelled costs based on future clinics being run by a band 7 HCP. The cost listed is per patient assuming eight patients seen during a 4-hour clinic with 2 hours of administration time. The HCP wages are based on the 2018 NHS AfC scales (midnodal point) and NHS Employers Pay and Conditions Circular March 2018.
The national tariff for a respiratory sleep study is $£ 408^{11}$ - this nominally includes the operating cost of the device and clinician reporting time. The unit cost for each device will vary between trusts and depend on the device and contractual agreement with the device manufacturer. We used the same device, within both the hospital and community pathways-hence this cost has not been included in our final calculations.

MDT costs were calculated based on the clinicians present and their hourly wage according to AfC and are calculated per patient based on six patients discussed per hour. The hospital MDT involves a consultant respiratory physician, two band 7 allied health professionals (AHPs) and band 4 AHP. The community MDT was undertaken with a consultant respiratory physician and band 7 AHP. MDT administrative cost was calculated in a similar manner.

Table 4 Cost estimates per patient for the community-based and hospital-based diagnostic pathways

\begin{tabular}{llll}
\hline & & Cost per patient & \\
\cline { 3 - 4 } Expense & & Hospital pathway & Outreach pathway \\
\hline Referral triage time & & $£ 3.44$ & $£ 3.44$ \\
Sleep study & Description & $2017 / 2018$ national tariff & $2017 / 2018$ national tariff \\
& Cost & $£ 408$ & $£ 408$ \\
Clinic appointment & Description & $2017 / 2018$ national tariff cost & 4-hour community clinic run by band 7+2 \\
& & for 1 new OPA and 1 F/U OPA & hour admin. using AfC (midnodal) \\
MDT & Total cost/patient & $£ 302$ & $£ 14.87$ \\
MDT administrative costs & Cost & $£ 16.58$ & $£ 11.05$ \\
& Description & 15 min/patient band 4 & 15 min/patient band 7 \\
& Cost & $£ 2.79$ & $£ 4.95$ \\
\hline
\end{tabular}

For the community clinic, costs are calculated based on eight patients being seen per clinic. Hospital clinic costs are calculated based on our data demonstrating an average two attendances (one new and one follow-up). ${ }^{11}$

AfC, Agenda for Change; F/U, follow-up appointment; MDT, multidisciplinary team; OPA, outpatient appointment. 


\section{DISCUSSION}

Our results demonstrate that a community-based approach to diagnosing OSA with virtual support from a hospital specialist team can result in significant reductions in waiting times, is favoured by patients and clinicians, and has the potential to achieve considerable costsavings. By undertaking an initial assessment and investigation in the community, only those patients who require treatment and review will be booked into secondary care clinics thus avoiding 'unnecessary' appointments. Patients with a normal sleep study and sleep disorders questionnaire which do not highlight any cause for concern will continue to receive care from their GP. This pathway can therefore reduce the number of unnecessary secondary care appointments which are not materially adding to patient care. In turn, this can reduce secondary care clinic waiting times for those who do require specialist input. For the patients, this means receiving care in a timely fashion, closer to home; and for hospital trusts, this can result in further cost-savings through streamlining clinics and referral pathways. We appreciate that an additional time resource will be required initially when setting up this service, however, we do not envisage the virtual MDT to generate significant extra work for the specialist and, as is our experience, it can sit alongside an existing MDT. The introduction of this community pathway should actually allow for a reduction in secondary care clinical workload which will subsequently allow for reallocation of the clinician's time to further devote to virtual MDT and consult.

The pathway closely aligns with STP (Sustainability and Transformation Partnership) plans of delivering care closer to home for patients and also with the NHS recently published 10-year priorities of greater integration with NHS organisations working closer with their local partners and reducing reliance on hospitals. ${ }^{9}$ Furthermore, delivery of diagnostics within a community setting will increase visibility and awareness of OSA both among patients and primary care physicians. The importance of the latter has recently been highlighted by surveys of primary care physicians in North Africa, the Middle East and Malaysia concluding that more work is required to support knowledge development and build confidence among these clinicians in recognising and supporting patients with OSA. ${ }^{12}$ While there does not appear any similar work conducted in the UK and although this was not something we assessed during this pilot, the increased exposure to OSA that this pathway offers might facilitate this process and also provide opportunity to deliver training within each hub locality.

Looking ahead, we hope to extend reach of the clinic beyond general practice such that referral to the pathway can be made by allied HCPs working within relevant community settings such as weight management services, dietetic and therapy departments. This might mediate wider economic ramifications by helping to access some of the 'missing million cases.' In their health economic analysis, the BLF estimates a $£ 55$ million saving for the
NHS with a gain of 40000 quality-adjusted life years annually and prevention of an additional 40000 motor vehicle accidents each year if all those with moderatesevere OSA were diagnosed and treated. ${ }^{7}$

Our findings build on previous studies which have demonstrated cost-effectiveness and equivalent rates of continuous positive airway pressure compliance with community-based models of care for patients with OSA when compared with hospital-based sleep units. ${ }^{14-16}$ However, these studies have either focused on the role of primary care in the ongoing follow-up of patients who have already been diagnosed with OSA in a hospital sleep unit $^{15}$; selecting only those with high probability for OSA syndrome ${ }^{14}$ or have evaluated a community-based approach to diagnosis which requires HCPs to conduct a home visit. ${ }^{16}$

In comparison, our pathway is the first to be undertaken within the UK NHS system, accepting all referrals for patients who may have a diagnosis of OSA and compares the costs directly associated with the diagnostic stage of OSA within a community (primary care) versus hospital setting. Furthermore, by using the existing primary care infrastructure, we did not require patients to be visited at home by an HCP; instead they were able to attend a GP practice within their local neighbourhood. To our knowledge, our approach is also the first to involve virtual secondary care-based multidisciplinary input to support the diagnostic process in the community. This recognises that the diagnosis of sleep disordered breathing requires clinical experience and expertise with a risk of missing some of the nuances if the whole process is transferred to primary care. This early support from the hospital-based team is a feature which was particularly welcomed by the GPs.

\section{Limitations}

The community outreach pathway was instituted within a borough with pre-existing strong collaborative working practices between constituent GPs. This method of working might have contributed to the successful outcomes of this pathway and might not have been observed to the same extent if the pathway was introduced in an alternative borough. However, enhanced collaboration and sharing of services in 'primary care networks' represents an important strategy of the NHS Long Term Plan and thus is likely representative of future service delivery and organisation across all of primary care.

When comparing the baseline characteristics between the two patient groups, there are significant differences in age, body mass index, smoking status, presence of hypercholesterolaemia and ESS. However, the groups are matched for sex and other comorbidities. This heterogeneity between the two groups likely reflects the fact that the community pathway, by virtue of it being a pilot, only drew from the population residing in one catchment neighbourhood versus the retrospective sample conducted of hospital outpatients which received referrals from across the entire catchment area. While these 
differences exist and might well account for some of the differences in final diagnoses between the groups, they are unlikely to affect the pathway process and cost outcomes which we have described in this paper.

For the purposes of this pilot, the community outreach clinic located at the hub practice was run by a sleep and ventilation clinical fellow with experience in secondary care sleep and ventilation medicine clinics. We anticipate that when this model of care is delivered beyond the pilot setting, the hub clinics will be run by an experienced band 7 HCP with adequate training in the provision of sleep medicine care and diagnostics. We have therefore modelled our costs based on this assumption. Further, premises and infrastructure costs were not incurred during our study as these were provided for free by the hub practice. However, we do not envisage that these will represent a significant outlay especially as there is a substantial appetite among primary care physicians to embed and spread this pathway further. Additionally, our cost analysis did not include an assessment of cost effectiveness but we plan to undertake this prospectively following wider dissemination of the pathway.

While our findings are encouraging, we acknowledge that we have only evaluated the diagnostic phase of OSA. We recognise that treatment and follow-up represent a significant workload burden for secondary care and we are therefore working with our local commissioners to establish this pathway across the STP and Integrated Care System footprint with a second phase planned to evaluate feasibility of treatment and follow-up.

\section{CONCLUSION}

Our data demonstrate that a community-based OSA diagnostic pathway with virtual specialist input can improve wait times, reduce hospital attendances, enhance both patient and GP experience of the sleep and ventilation service, and has the potential to generate cost-savings. It embraces the NHS long-term vision of greater integration between care providers and helps bridge some of the traditional divides between community and hospital care thereby providing more joined-up care. The simplicity in its design will facilitate its implementation beyond our local footprint, allow eventual incorporation of a treatment and follow-up arm and can also serve as a model for other diagnostic processes traditionally undertaken in secondary care.

Contributors ND, TA, SMorgan, SMandal conceived and designed the community OSA pathway. All authors contributed to the implementation of the pathway. ND, SKM, FL and SMandal collected and interpreted data. ND and SMandal took lead in writing the manuscript with input from all authors. All authors approved the final version of the manuscript for submission. SMandal supervised the project.

Funding WatchPAT devices supplied without charge by Itamar Medical.

Competing interests None declared.

Patient consent for publication Not required.

Provenance and peer review Not commissioned; externally peer reviewed.

Data availability statement All data relevant to the study are included in the article or uploaded as supplemental information.
Supplemental material This content has been supplied by the author(s). It has not been vetted by BMJ Publishing Group Limited (BMJ) and may not have been peer-reviewed. Any opinions or recommendations discussed are solely those of the author(s) and are not endorsed by BMJ. BMJ disclaims all liability and responsibility arising from any reliance placed on the content. Where the content includes any translated material, BMJ does not warrant the accuracy and reliability of the translations (including but not limited to local regulations, clinical guidelines, terminology, drug names and drug dosages), and is not responsible for any error and/or omissions arising from translation and adaptation or otherwise.

Open access This is an open access article distributed in accordance with the Creative Commons Attribution Non Commercial (CC BY-NC 4.0) license, which permits others to distribute, remix, adapt, build upon this work non-commercially, and license their derivative works on different terms, provided the original work is properly cited, appropriate credit is given, any changes made indicated, and the use is non-commercial. See: http://creativecommons.org/licenses/by-nc/4.0/.

ORCID iDs

Nikesh Devani http://orcid.org/0000-0001-5012-4165

Stephanie K Mansell http://orcid.org/0000-0002-2806-380X

Swapna Mandal http://orcid.org/0000-0002-2232-5880

\section{REFERENCES}

1 Young T, Palta M, Dempsey J, et al. The occurrence of sleepdisordered breathing among middle-aged adults. $N$ Engl $\mathrm{J}$ Med 1993;328:1230-5.

2 Benjafield AV, Ayas NT, Eastwood PR, et al. Estimation of the global prevalence and burden of obstructive sleep apnoea: a literaturebased analysis. Lancet Respir Med 2019;7:9-12.

3 McNicholas WT, Bonsigore MR, Bonsignore MR, et al. Sleep apnoea as an independent risk factor for cardiovascular disease: current evidence, basic mechanisms and research priorities. Eur Respir J 2007;29:156-78.

4 Kent BD, Grote L, Ryan S, et al. Diabetes mellitus prevalence and control in sleep-disordered breathing: the European sleep apnea cohort (ESADA) study. Chest 2014;146:982-90.

5 Terán-Santos J, Jimenez-Gomez A, Cordero-Guevara J. The association between sleep apnea and the risk of traffic accidents. $N$ Engl J Med Overseas Ed 1999;340:847-51.

6 George CF. Reduction in motor vehicle collisions following treatment of sleep apnoea with nasal CPAP. Thorax 2001;56:508-12.

7 Rejón-Parrilla JC, Garau M, Sussex J. Obstructive Sleep Apnoea Health Economics Report - Consulting Report for the British Lung Foundation, 2014. Available: https://www.blf.org.uk/sites/default/ files/OHE-OSA-health-economics-report-FINAL-v2.pdf

8 Department of Health. The NHS plan : a plan for investment : a plan for reform : presented to parliament by the Secretary of State for Health by command of Her Majesty, July 2000. C ; 4818-I 2000;:144.

9 NHS England. The NHS long term plan - a summary, 2019: 2.

10 Ogrinc G, Davies L, Goodman D, et al. Squire 2.0 (standards for quality improvement reporting excellence): revised publication guidelines from a detailed consensus process. BMJ Qual Saf 2016;25:986-92.

11 NHS Improvement. National tariff 2017/19, 2019. Available: https:// improvement.nhs.uk/resources/developing-the-national-tariff/ [Accessed 25 Jan 2020].

12 Al-Khafaji H, Bilgay IB, Tamim H, et al. Knowledge and attitude of primary care physicians towards obstructive sleep apnea in the middle East and North Africa region. Sleep Breath 2020. doi:10.1007/s11325-020-02137-7. [Epub ahead of print: 01 Jul 2020].

13 Devaraj NK. Knowledge, attitude, and practice regarding obstructive sleep apnea among primary care physicians. Sleep Breath 2020. doi:10.1007/s11325-020-02040-1. [Epub ahead of print: 24 Feb 2020].

14 Andreu AL, Chiner E, Sancho-Chust JN, et al. Effect of an ambulatory diagnostic and treatment programme in patients with sleep apnoea. Eur Respir J 2012;39:305-12.

15 Sánchez-de-la-Torre M, Nadal N, Cortijo A, et al. Role of primary care in the follow-up of patients with obstructive sleep apnoea undergoing CPAP treatment: a randomised controlled trial. Thorax 2015;70:346-52.

16 Chai-Coetzer CL, Antic NA, Rowland LS, et al. A simplified model of screening questionnaire and home monitoring for obstructive sleep apnoea in primary care. Thorax 2011;66:213-9. 